\title{
Media Information Dissemination Model of Wireless Networks Using Deep Residual Network
}

\author{
Xiaojing Lv (iD) and Dongphil Chun \\ Graduate School of Management of Technology, Pukyong National University, Busan 48547, Republic of Korea \\ Correspondence should be addressed to Xiaojing Lv; lvxiaojingself@mail.sdufe.edu.cn
}

Received 18 April 2021; Revised 25 May 2021; Accepted 25 June 2021; Published 2 July 2021

Academic Editor: Fazlullah Khan

Copyright (c) 2021 Xiaojing Lv and Dongphil Chun. This is an open access article distributed under the Creative Commons Attribution License, which permits unrestricted use, distribution, and reproduction in any medium, provided the original work is properly cited.

\begin{abstract}
Information dissemination and its prediction in wireless networks is a challenging task. Researchers have studied the prediction process of media information dissemination in wireless networks using various methods. In this paper, we analyze information dissemination in wireless networks using a deep residual network model. In the proposed model, the relative weight of nodes and the dissemination probability of media information in wireless networks are obtained. The obtained information is the inputs into the deep residual network as features. The convolution feature extractor is used to obtain the details of the input features. Finally, the propagation information is classified according to the extracted features through the full connection layer. We have used the SELU activation function to optimize the deep residual network. In this way, a complete media information dissemination prediction of wireless networks is obtained. The simulation results show that the proposed model has fast convergence and a low bit error rate of information dissemination. It reflects the characteristics of media information dissemination in a wireless network in real-time applications. The results show accurate prediction of media information dissemination in wireless networks.
\end{abstract}

\section{Introduction}

Media refers to a carrier used to spread all kinds of information. It includes all the materials and tools used in the process of information dissemination. The information dissemination from the sender to the receiver is transmitted through the different material tools [1]. The development of media information can be divided into several stages, for example, gesture media that realizes the expression and dissemination of information through gesture or beacon fire. The paper letters and text media are used to complete the dissemination of information through reading, television, and radio. Other equipment media, which complete the dissemination of information through direct screen, content, and language. There is also a kind of wireless network media, which is produced with the continuous progress of network technology. It has significant advantages, regardless of time constraints and space constraints, and can convey information at any time [2]. Intelligent devices have become the necessities of people's lives in modern society. The audience can view mobile phones anytime and anywhere, and there are various forms of advertising. Therefore, wireless media is developing with the trend. Various industries are vigorously carrying out wireless media, through the advertising value of wireless media, to achieve sales and other purposes [3]. Media information dissemination in a wireless network has a very important application value in advertising, event marketing, public opinion monitoring and guidance, influence quantification, network communication, and other aspects. With the increasing popularity and scale of social networks, social network-related research has attracted most researchers' attention. In these studies, how to describe the dissemination behavior of information in social networks revealing their characteristics and dissemination rules has very important theoretical research and practical application value and one of the current research hotspots. The residual network (RESNET) is a convolution neural network proposed by four scholars from Microsoft Research. It can improve the efficiency of information dissemination by adding directly connected edges to the nonlinear convolution layer [4]. 
Scholars have carried out a lot of research work on network communication and its applications. These include the spread of computer viruses on computer networks and the spread of rumors in social networks. Similarly, the spread of infectious diseases among people and information in online communities, blogs, etc. is also included. These can be regarded as disseminating certain information laws [5]. The dissemination process of information in the real network is affected by the network status of information publishers and their behavior. Whether information can spread from one node to another and make the latter become the disseminator is related to the influence of the disseminator and the latter's receptivity. For example, in [6], Zhang and He designed an information dissemination model based on the interaction between neighbor nodes and the improved probability. In this model, the false immune node was introduced into the basic epidemic dynamics model. Three dissemination probability functions were defined to complete the inference of information dissemination efficiency. In [7], the information dissemination model based on behavior analysis was designed by Han. The establishment of the model was based on the information published by information publishers, information receivers, and information dissemination platforms that would affect users' forwarding behavior. On this basis, features were extracted to design an information dissemination prediction model. However, in media information dissemination prediction, the two models $[6,7]$ have no relevant results. In these models, there is a big difference between the fitting results and the actual results. Therefore, in this paper, we analyze the information dissemination model in wireless networks using a deep residual network. The proposed model is used to realize the prediction of media information dissemination in wireless networks.

The rest of the paper is organized as follows. In Section 2, the proposed model is discussed in detail. The results and discussion are given in Section 3, followed by the conclusion in Section 4.

\section{Media Information Dissemination Model of Wireless Network Based on Deep Residual Network Technology}

This section discusses the probability acquisition, information dissemination model, and residual network for media information dissemination.

\footnotetext{
2.1. Probability Acquisition of Media Information Dissemination in Wireless Network. In the communication process of media information in a wireless network, the mutual influence of the node itself and neighbor nodes is very important to information forwarding. We provide some definitions here to make the proposed model easily understandable. In these definitions, the term $g$ represents a node in the network, and the value is the number of the network node. The node influence parameter of the network is defined as follows.
}

Definition 1. Degree $k(g)$ of a node, i.e., the number of neighbors of a node $g$, such as $k(1)=6$.

Definition 2. A neighbor set $\Gamma(g)$ of node, that is, the set of all neighbor nodes of node $g$, such as $\Gamma(1)=\{2,4,5,6,9,10\}$.

Definition 3. Node influence weight $f\left(g_{i}, g_{j}\right)$. Let $g_{i} \in \Gamma\left(g_{j}\right)$ be a neighbor of $g_{i}$; then, the influence of $g_{i}$ on $g_{j}$ is defined as

$$
f\left(g_{i}, g_{j}\right)=\frac{k\left(g_{i}\right)}{\sum_{g_{l} \in \Gamma\left(g_{j}\right)} k\left(g_{l}\right)},
$$

where the sum of degrees of all neighbors of a point $g_{j}$ is $\sum_{g_{l} \in \Gamma\left(g_{j}\right)} k\left(g_{l}\right)$. The greater the degree $k\left(g_{i}\right)$ of a node $g_{i}$ is, the greater the influence $f\left(g_{i}, g_{j}\right)$ on a node $g_{j}$ is.

Definition 4. The relative influence weight $\mathrm{Rw}\left(g_{i}, g_{j}\right)$ of a node $g_{i}$ on a node $g_{j}$. Let nodes $g_{j}$ and $g_{j}$ be neighbors to each other. Combined with Definition 3 , the relative influence weight $\operatorname{Rw}\left(g_{i}, g_{j}\right)$ is defined as

$$
\operatorname{Rw}\left(g_{i}, g_{j}\right)=2 \frac{f\left(g_{i}, g_{j}\right)}{f\left(g_{i}, g_{j}\right)+f\left(g_{j}, g_{i}\right)}
$$

It should be noted that $\operatorname{Rw}\left(g_{i}, g_{j}\right)$ is usually not equal to $\operatorname{Rw}\left(g_{j}, g_{i}\right)$. It is determined by the relative authority of nodes $g_{j}$ and $g_{j}$. The role of $\operatorname{Rw}\left(g_{i}, g_{j}\right)$ is to readjust the weight of the sides connecting $g_{j}$ and $g_{j}$ according to their relative positions [8].

After the node $g_{S}$ contacts the dissemination node $g_{I}$, regardless of the status of both nodes, the probability of the node $g_{S}$ transforming into a dissemination node is fixed, i.e., $p_{1}=\lambda$. It does not conform to the characteristics of real media information dissemination in a wireless network [9]. In the RWSIR model considering the relative influence, the information dissemination is affected by the type of dissemination path. The transformation probability will be reduced or enlarged to $\operatorname{Rw}\left(g_{I}, g_{S}\right) \lambda$ times of the original. The dissemination probabilities of different paths are shown in Figure 1.

The probability $p_{1}$ of information on the $P_{a}$ path is enlarged sharply. In contrast, that on the $P_{b}$ path is reduced sharply, while that on $P_{c}$ and $P_{d}$ paths is not obvious. The model reflects that in information dissemination when the authority of two nodes is not equal $\left(P_{a}\right.$ and $\left.P_{b}\right)$, the probability of dissemination $p_{1}$ depends on the authority $\operatorname{Rw}\left(g_{I}, g_{S}\right) \lambda$ rather than the popularity $\lambda$. It is easy for the nodes to persuade the nodes with less authority to accept their views, while it is more difficult to do the opposite [10]; when the difference of authority between the two nodes is small, the model has little effect on it. The propagation probability $p_{1}$ mainly depends on the information's popularity $\lambda$ rather than the authority $\operatorname{Rw}\left(g_{I}, g_{S}\right) \lambda$.

Based on the model, combined with the relative weight $R w\left(g_{I}, g_{S}\right)$ of nodes and the information dissemination characteristics of social networks, the basic dissemination probability $\lambda$ is given. At the same time, $p_{1}$ as a probability cannot exceed 1 , and the value of $\operatorname{Rw}\left(g_{I}, g_{S}\right)$ may exceed 1 

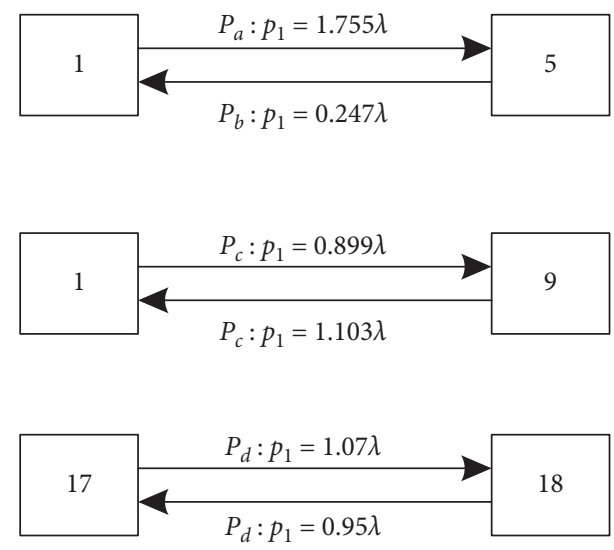

Figure 1: Dissemination probabilities of different paths.

under certain conditions. If it exceeds 1 , we specify $p_{1}=1$ that the healthy node will become a dissemination node [11]. Let $p_{1}\left(g_{I}, g_{S}\right)$ be the dissemination probability of dissemination node $g_{I}$ to healthy node $g_{S}$, which can be expressed as

$$
p_{1}\left(g_{I}, g_{S}\right)= \begin{cases}\operatorname{Rw}\left(g_{I}, g_{S}\right) \lambda, & \operatorname{Rw}\left(g_{I}, g_{S}\right) \lambda<1, \\ 1, & \operatorname{Rw}\left(g_{I}, g_{S}\right) \lambda \geq 1 .\end{cases}
$$

2.2. Dissemination Model Based on Deep Residual Network. Depth feature synthesis is a method of constructing feature engineering, which can construct new and deep features from single or multiple data tables through field conversion and aggregation. Unlike other feature extraction methods such as convolution, the process of depth feature synthesis is visible, and the new features constructed have practical significance [12]. The given data can be split into two-tier entity sets. Depth feature synthesis creates features by applying the obtained dissemination probability to the entity relationships in the entity set.

A deep convolution neural network is mainly composed of a data input layer, hidden layer, and output layer. The hidden layer contains a multilevel neural network, which is mainly built by the combination of convolution layer, pooling layer, and full connection layer. A convolution feature extractor is used to obtain the details of the input dissemination probability. Finally, fully connected (FC) layers classify the dissemination information according to the extracted dissemination features to complete the dissemination prediction, as shown in Figure 2.

The network performance can be improved using a deep convolutional neural network. It is done by using nonlinear activation functions and batch normalization (BN) layers. In the hidden layer, each neural unit of each layer is connected with all neural units of the next layer, and all connections have a weight. In the network training process, for the output results of each layer, the degree of classification error needs to be measured by loss function calculation. The weight of each layer is updated by gradient descent [13].



Figure 2: Convolutional neural network structure.

In a convolutional neural network, for an $L$-layer convolutional, the gradient calculation formula of its training network is as follows:

$$
\frac{\partial L_{\text {loss }}}{\partial x_{1}}=\frac{\partial H_{n}\left(x_{L_{n}}, W_{L_{n}}, b_{L_{n}}\right)}{\partial L_{L}} \times \cdots \times \frac{\partial H_{2}\left(x_{L_{2}}, W_{L_{2}}, b_{L_{2}}\right)}{\partial x_{1}} .
$$

The input and weight matrix of the calculation loss and the $n$-th layer is $L_{\text {loss }}, x_{L_{n}}$, and $W_{L_{n}}$, respectively, and the offset of this layer and the calculation loss of the $n$-th layer are $b_{L_{n}}$ and $H_{n}\left(x_{L_{n}}, W_{L_{n}}, b_{L_{n}}\right)$.

It can be seen from the aforementioned formula that with the increase of network depth, the gradient amplitude will decrease/increase sharply. It results in the weight update of shallow neurons too slow/fast via gradient disappearance/ gradient explosion and the performance degradation of the model. Therefore, He and Sun proposed optimizing the deep learning network by residual mapping. They form residual blocks by skipping the convolution layer by using a jump connection. It solved network model degradation to a great extent and greatly improved the training efficiency [14]. The model framework is shown in Figure 3.

On the left side of Figure 3 is the residual unit constructed in this study. The input and output dimensions of each residual unit are the same. The $\mathrm{BN}$ layer uses its average value $\mu_{b}$ and variance $\sigma_{b}^{2}$. The input of the activation function is standardized, and then the batch normalization results are scaled by stretch parameters and offset parameters to make the data more dispersed and more uniform in space. The activation function is used to extract the complex features of the data and increase the nonlinear relationship of each layer network [15]. Adding the batch normalization results of the 




FIgURE 3: Deep residual network framework.

input of the residual cell and the output of the second convolution in a residual cell, the original mapping function is expressed as $H\left(x_{k}\right)+x_{k}$ breaking through the network degradation problem caused by increased network depth. The gradient calculation formula in the depth network model is as follows:

$$
\frac{\partial L_{\text {loss }}}{\partial x_{1}}=\left(1+\frac{\partial H_{n}\left(x_{L_{n}}, W_{L_{n}}, b_{L_{n}}\right)}{\partial x_{L}}\right) \times \cdots \times\left(1+\frac{\partial H_{2}\left(x_{L_{2}}, W_{L_{2}}, b_{L_{2}}\right)}{\partial x_{1}}\right) .
$$

The deep residual network updates the parameters of the input data directly connected to the overlay layer while updating the parameters of the overlay layer. The low-order disease features obtained by the shallow network and the high-order disease features extracted by the deep network are combined. The gradient can flow directly from the latter layer to the former layer through the identical activation function, making the residual network have faster convergence speed and stronger feature expression ability than traditional convolutional neural networks [16].

In order to improve the efficiency of information dissemination, adding directly connected edges to the nonlinear convolution layer is proposed. Suppose that a nonlinear element (which can be one or more convolution layers) $f(x, \theta)$ is expected to approximate an objective function $h(x)$ in a deep network. The objective function is divided into two parts: identity function $x$ and residual function $h(x)-x$, namely,

$$
h(x)=x(h(x)-x) .
$$

According to the general approximation theorem, a nonlinear element composed of a neural network has enough ability to approximate the original objective function or residual function. However, the latter is easier to learn in practice [17]. Therefore, the original optimization problem can be converted to let the nonlinear element $f(x, \theta)$ approximate the residual function $h(x)-x$ and use $f(x, \theta)+x$ to approximate $h(x)$.

In the process of building the model, the dissemination path loss $L_{b}$ is used as the training tag. The forward reasoning process of the model is as follows:

$$
\left\{\begin{array}{l}
Y=P_{t}-\operatorname{RSRP} \\
X_{\text {std }}=\sigma_{X}(X), Y_{\text {std }}=\sigma_{Y}(Y), \\
\hat{Y}=\operatorname{Resnet}\left(X_{\text {std }}\right) \\
\text { loss }=\operatorname{MSE}\left(\hat{Y}, Y_{\text {std }}\right)
\end{array}\right.
$$

2.3. Residual Network Improvement. After the neural network is activated, it can solve the problem of a nonlinear function. The ReLU activation function is one of the best. From a computational point of view, ReLU calculation is simple, and only a threshold is needed to calculate the activation value. Secondly, the unsaturated property of ReLU can effectively solve the problem of gradient disappearance to provide a relatively wide activation boundary [18]. Finally, the unilateral suppression of ReLU provides the sparse expression ability of the network. However, ReLU still has its limitations. The formula is as follows: 


$$
\operatorname{ReLU}(x)= \begin{cases}x, & \text { if } x>0 \\ 0, & \text { if } x \leq 0\end{cases}
$$

According to the formula, this function is directly set to 0 on the negative half axis. The negative gradient will not be activated when passing through the ReLU unit, resulting in the death of the neuron [19]. Secondly, when the positive half axis derivative of the ReLU function is constant to 1 , the gradient will disappear when it passes through the function.

SELU activation function is generated by solving the above problems. As shown in formula (9), the positive half axis derivative of the SELU activation function is greater than 1. It can increase when the variance is too small and prevent the gradient from disappearing. In contrast, the negative half axis is no longer simply set to 0 , thus solving the problem of neuron death [20]. Its slope is relatively gentle, to ensure the unilateral inhibition advantage in rReLU.

$$
\operatorname{SELU}(x)= \begin{cases}x, & \text { if } x>0 \\ a e^{x}-\alpha, & \text { if } x \leq 0 \\ \lambda>1 . & \end{cases}
$$

A two-layer residual structure is adopted. The SELU activation function replaces the ReLU activation function, and the data is normalized in batches. The improved residual network structure is shown in Figure 4.

\section{Experimental Analysis}

The simulation experiment is designed by using MATLAB 7 to test the application performance of the proposed model in the realization of media information dissemination in a wireless network. The equalizer order of wireless network dissemination is 15 . The tap interval of the wireless communication channel is $0.35 \mathrm{~s}$, the channel impulse response strength is $100 \mathrm{~dB}$, and the linear modulation is achieved under the wireless network. The carrier frequency is $240 \mathrm{kbps}$. The received signal of the routing node is sampled at more than 2 times the baud rate, and the multipath width of the detection signal is taken as $T_{g}=200 \mathrm{~ms}$. The dataset contains four types of media information, namely, recruitment information, advertising and entertainment information, social information, and livelihood news information. The details are shown in Table 1.

Based on the verification set and training set in Table 1, 20-layer and 30-layer deep neural networks are constructed to test the accuracy of the proposed model to disseminate media information in the verification set. The results are shown in Figure 5.

According to the test results in Figure 5, when the number of network layers is fixed, with the increase of the number of iterations, the accuracy of information dissemination presents a small fluctuation, and different network layers have a great impact on the results of information dissemination. With the increase of network layers, the accuracy of information dissemination of a 30-layer network is lower than that of a 20-layer network. The results show that the depth residual network model has the problem of gradient vanishing when carrying out media information

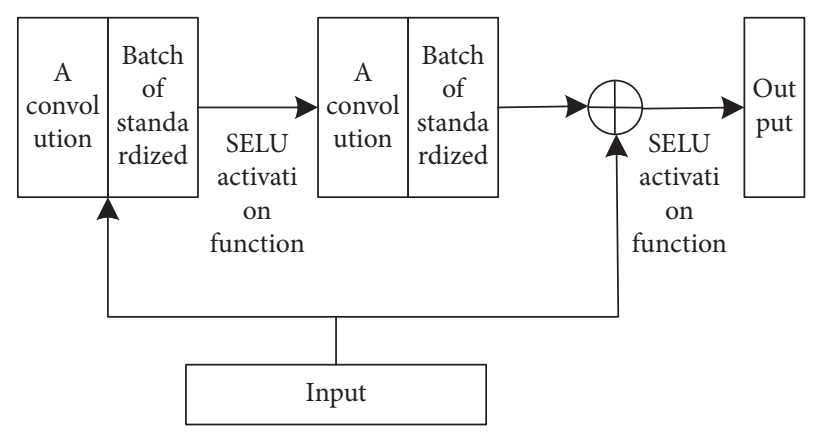

FIgURE 4: Modified residual structure.

dissemination. Therefore, this paper selects a 20-layer network structure to optimize it.

According to the test results in Figure 5, the SELU activation function is used to replace the ReLU activation function to optimize the network, test the test set, and analyze the accuracy of media information dissemination. The results are shown in Figure 6. At the same time, the best combination of parameters (minimum prediction point) is obtained, which is used for the real objective function (a classification error rate of validation set) to obtain the minimum observation value. Then, the proxy model containing the new minimum observation point is updated. The above steps are repeated to obtain the optimal combination of parameters. The average error of media information dissemination is obtained when the function is calculated for different times in the optimization process. The results are shown in Figure 7.

According to the test results in Figures 6 and 7, the convergence speed of the SELU activation function is better than that of the ReLU activation function. It can minimize the global search, avoid local optimization, and ensure the rapid convergence of the model. The average error of the minimum observed value and the minimum predicted value is the lowest in 16 calculations.

The coverage of the communication model in the process of communication is the main measurement standard. Therefore, the poor coverage recognition rate (PCRR) is selected as the index to measure the information dissemination of the proposed model. In communication, if we can effectively identify the poor coverage area, we can better help operators plan and optimize the network to improve the customer experience. PCRR is a very valuable evaluation index. The value of the poor coverage decision threshold $P_{\text {th }}$ studied in this paper is $-105 \mathrm{dBm}$. If the predicted or measured value of RSRP is less than $P_{\text {th }}$, it is weak coverage and marked as 1; if it is greater than or equal to $P_{\text {th }}$, it is nonpoor coverage and marked as 0 . According to the difference of poor coverage and nonpoor coverage between the predicted value and the measured value, the following parameters can be counted: true positive (TP): the real value is poor coverage, and the predicted value is also poor coverage; false positive (FP): the real value is nonpoor coverage, and the predicted value is poor coverage; false negative (FN): the real value is weak coverage, and the predicted value is nonpoor coverage; true positive (FP): the real value is 
TABLE 1: Information dataset information details.

\begin{tabular}{lccc}
\hline Information types & $\begin{array}{c}\text { Number of training set } \\
\text { messages }\end{array}$ & $\begin{array}{c}\text { Dataset/bar } \\
\text { The number of validation set } \\
\text { messages }\end{array}$ & The total number of messages \\
\hline $\begin{array}{l}\text { Recruitment information } \\
\text { Advertising entertainment }\end{array}$ & 5340 & 1590 & 6930 \\
information & 6030 & 1840 & 7870 \\
Social information & 3160 & 1900 & 5060 \\
People's livelihood news & 5690 & 940 & 6630 \\
\hline
\end{tabular}

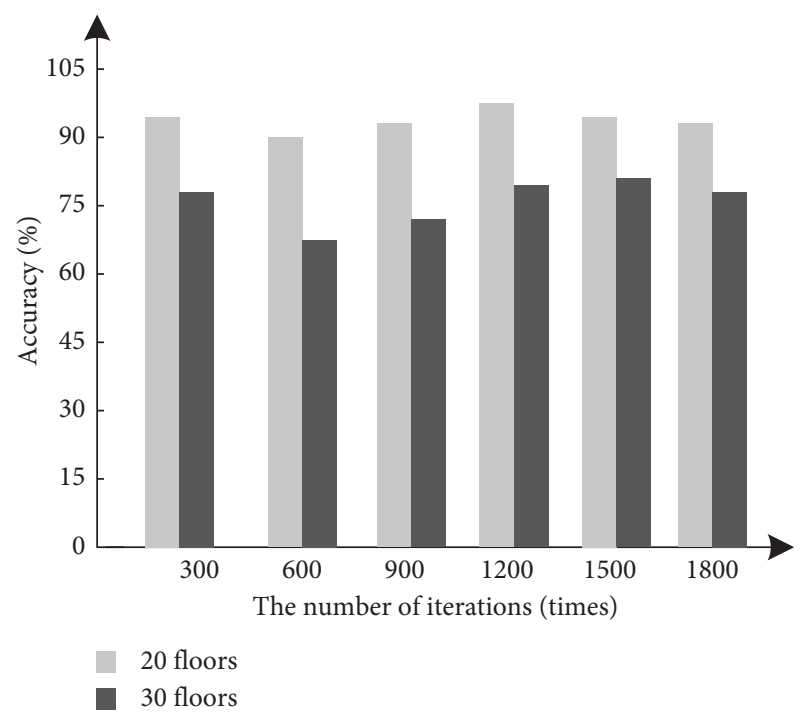

Figure 5: Test results of model information dissemination accuracy.

nonpoor coverage; true negative (TN): the real value is nonpoor coverage, and the predicted value is also nonpoor coverage.

The accuracy of PCRR is as follows: PCRR considers the target of precision and recalls comprehensively, and its calculation formula is as follows:

$$
\text { PCRR }=2 \times \frac{\text { precision } \times \text { recall }}{\text { precision }- \text { recall }},
$$

where precision can be understood as the probability that a grid with weak coverage is actually poor coverage and it is defined as

$$
\text { precision }=\frac{\mathrm{TP}}{\mathrm{TP}+\mathrm{FP}}
$$

Recall can be understood as the probability that the real result of a grid with weak coverage is predicted to be weak coverage and it is defined as

$$
\text { recall }=\frac{\mathrm{TP}}{\mathrm{TP}+\mathrm{FN}^{\circ}}
$$

The dataset is tested, and the change of weak coverage rate of average received power on the training set and test set is counted. The results are shown in Figure 8.

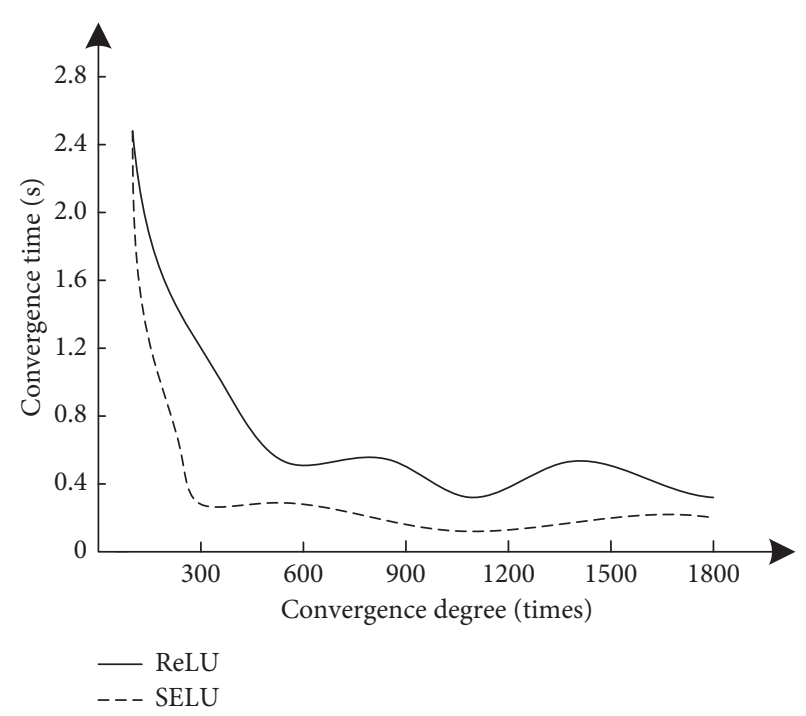

FIgURE 6: Test results of the convergence rate of the two functions.

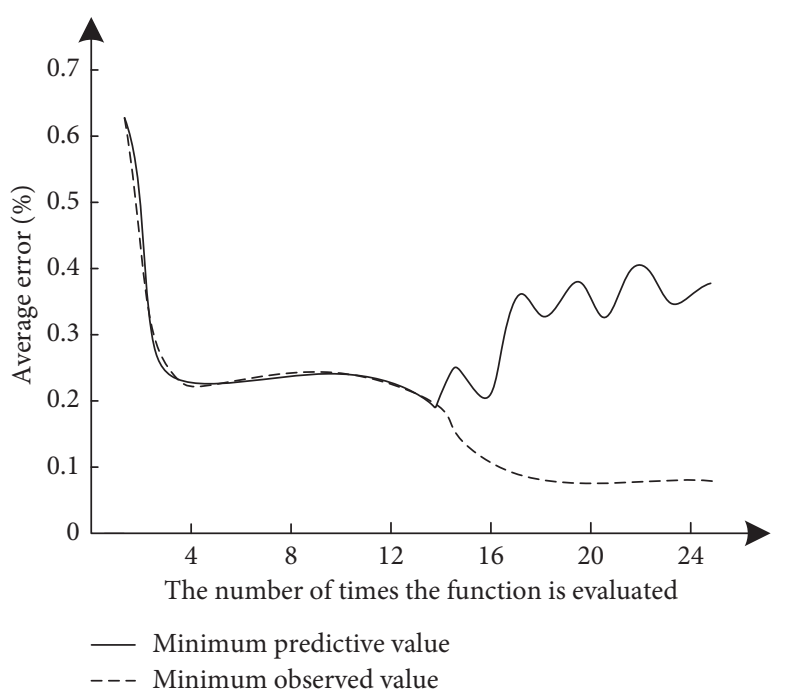

FIgURE 7: Test results of mean error of media information dissemination.

According to the test results in Figure 8, we can see that we have established a good regression model after training and achieved good results in the test set. After the weak coverage rate reaches the standard, the root mean square error on the test set is reduced to 8.15 (local) and 10.05 




(a)

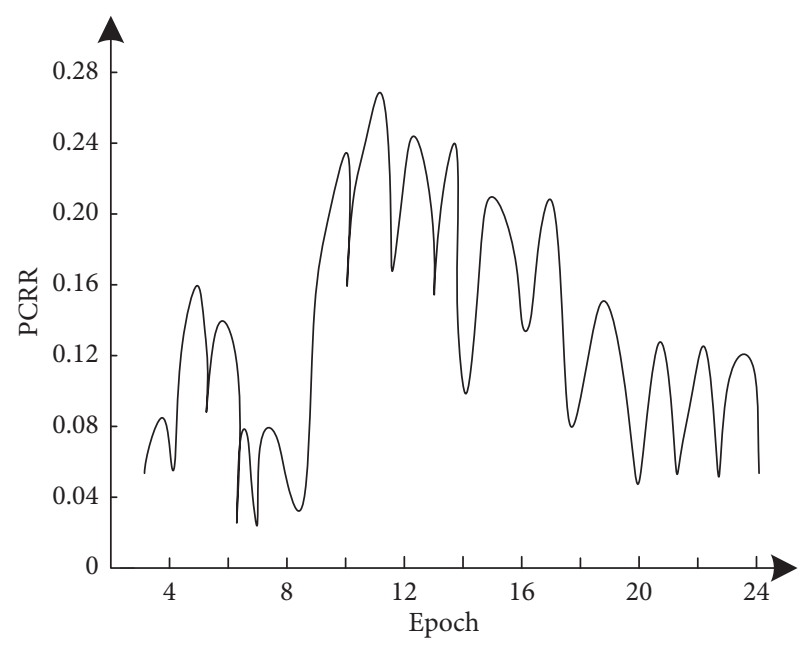

(b)

FIGURE 8: RMS error and weak coverage test results of the average received power of the model. (a) Loss results of the training set and test set. (b) Weak coverage test results for the test set.

(online). It proves that the information dissemination effect is good and the model performance is excellent.

In order to test the optimization effect of the proposed model, the impulse response characteristics of media information dissemination signals in a wireless network with different bandwidths are obtained. The dissemination signal frequency band is $4 \mathrm{kHz}$ and $6 \mathrm{kHz}$, respectively, before and after optimization, as shown in Figures 9 and 10.

According to the test results of Figures 9 and 10, before optimization, when the dissemination signal frequency band is $4 \mathrm{kHz}$ and $6 \mathrm{kHz}$, respectively, the collected signal is relatively scattered and fluctuates obviously, and the concentrated area is more affected by noise. After optimization processing, the output signal has good anti-interference performance, small fluctuation, high signal connectivity, and strong noise suppression ability. Therefore, the results show that, after optimization, it can more clearly describe the communication state of media information.

Based on the same verification set and test set, the forward reasoning matrix is constructed. The information dissemination model based on the interaction and improved probability between neighbor nodes in [6] and [7] and the information dissemination model based on behavior analysis are selected as the comparison models of the models in this paper. The accuracy of the prediction of four kinds of media information dissemination is counted. The results are shown in Figure 11.

According to the test results in Figure 11, the accuracy of the proposed model for four kinds of media information dissemination prediction is the best, which is significantly better than the two comparison models, and the accuracy is more than $97.4 \%$. Therefore, the proposed model has a good prediction effect of the law of media information dissemination in a wireless network. It can clearly and accurately depict the dissemination state of media information to ensure accurate information dissemination.

In order to measure the depiction of the proposed model for the law of media information dissemination in a wireless network, three models are used to carry out the fitting test for information dissemination, respectively. The fitting results of the three models are counted and compared with the actual results. The fitting conditions of the three models are compared, as shown in Table 2.

According to the test results in Table 2, the fitting results of the model in this paper are the closest to the actual results. The fitting results of the two comparative models are quite different from the actual results. In the ideal state, the peak value of the number of dissemination nodes of the model in [6] is the best. However, it is significantly different from the actual data. In the early stage of information dissemination, the increasing trend of the number of dissemination nodes of the model in [7] is lower than that of the proposed model. However, with the continuous dissemination of information, the reference model [7] shows a significant downward trend. The results show that in the actual communication application process, each individual's media message will be affected in different degrees, which will affect the communication process. Therefore, the proposed model considers the information dissemination of node attitude, which is more in line with the dissemination law of the real network and can achieve better fitting results.

In order to measure the dissemination performance of the models, in this paper, the output bit error rate of the three models in the process of information dissemination for the four media information dissemination channels is tested. The obtained results and test comparison are shown in Figure 12. 


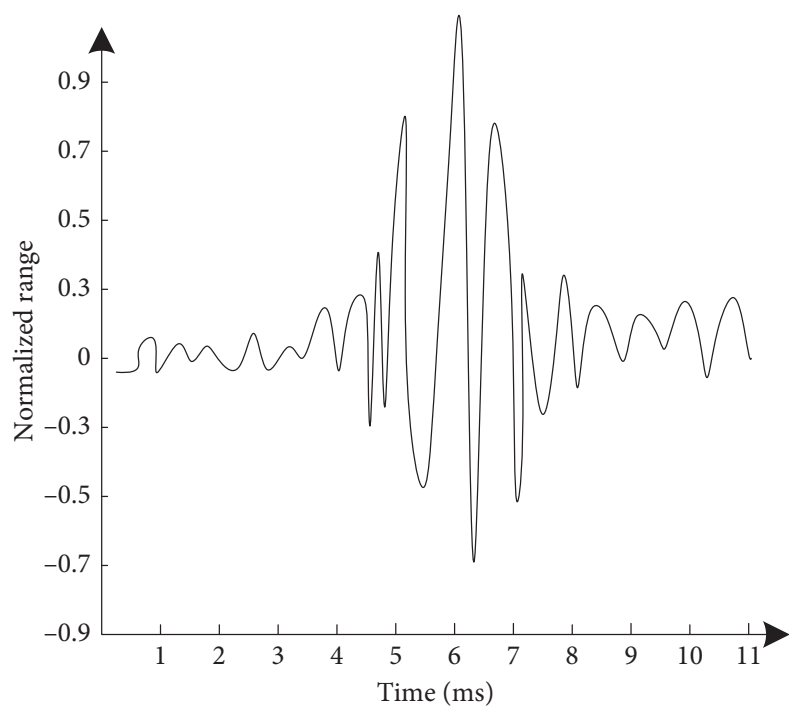

(a)

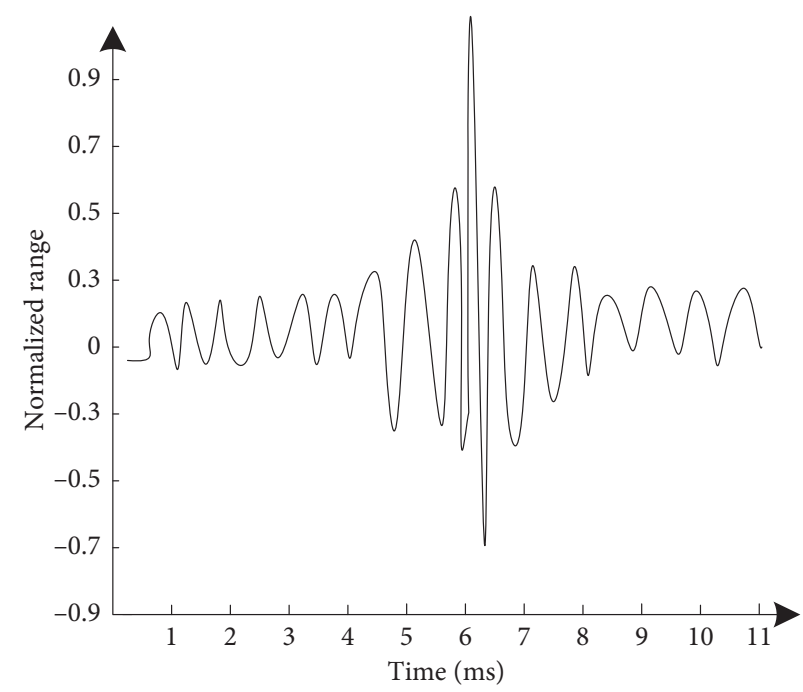

(b)

FIGURE 9: Eigenvalues of the impulse response of propagated signals before model optimization. (a) Before optimization, the frequency band of the transmitted signal is $4 \mathrm{kHz}$, respectively. (b) Before optimization, the frequency band of the transmitted signal is $6 \mathrm{kHz}$, respectively.

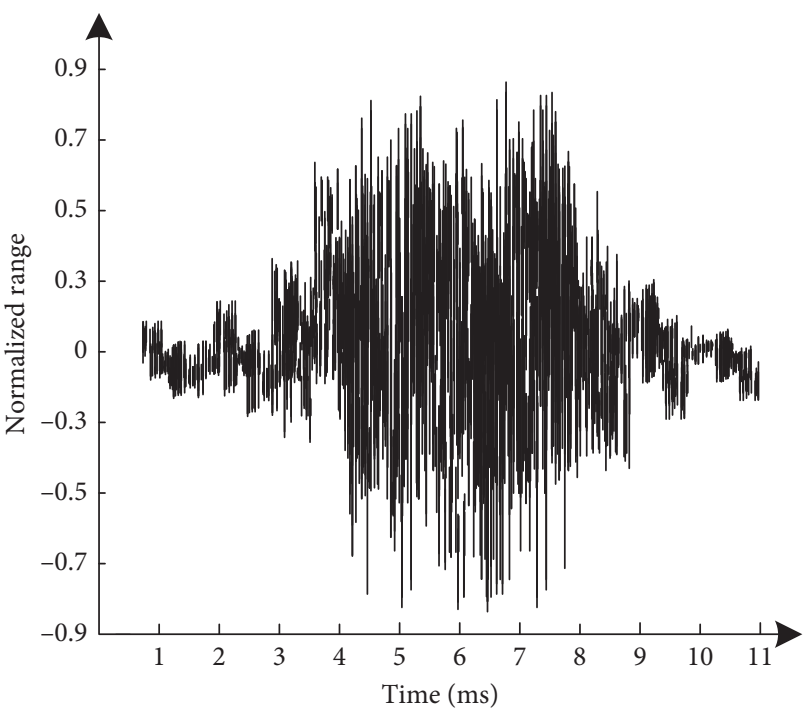

(a)

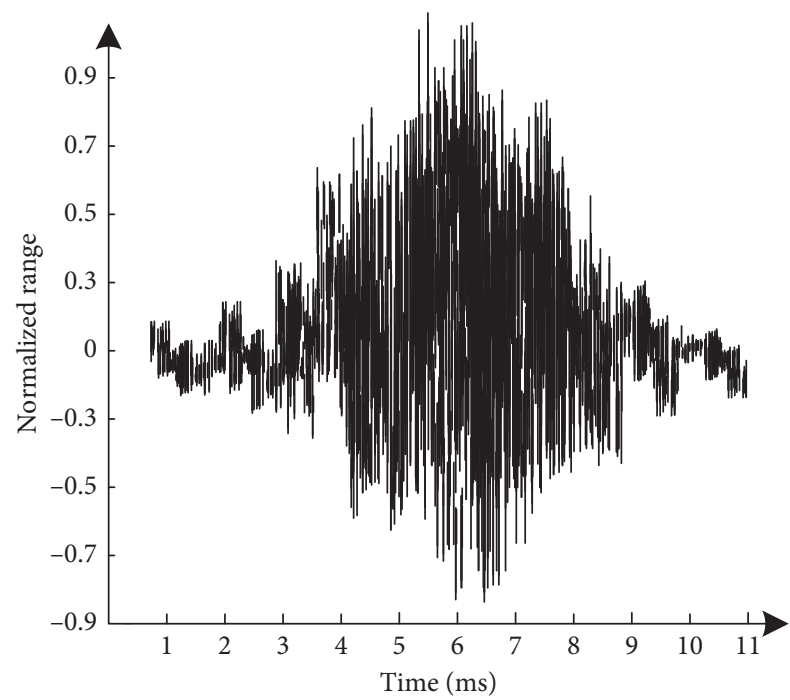

(b)

Figure 10: Impulse response characteristic quantity of optimized media information dissemination signal in the wireless network. (a) After optimization, the frequency band of the wireless network media information dissemination signal is $4 \mathrm{kHz}$ impulse response characteristic quantity, respectively. (b) After optimization, the frequency band of the wireless network media information dissemination signal is $6 \mathrm{kHz}$ impulse response characteristic quantity, respectively.

According to the test results in Figure 12, the bit error rate of the proposed model is the lowest for the four media information dissemination channels. It is significantly lower than the output bit error rate of the two comparison models. Therefore, the symbol distortion of the model is less than $4.2 \%$. 


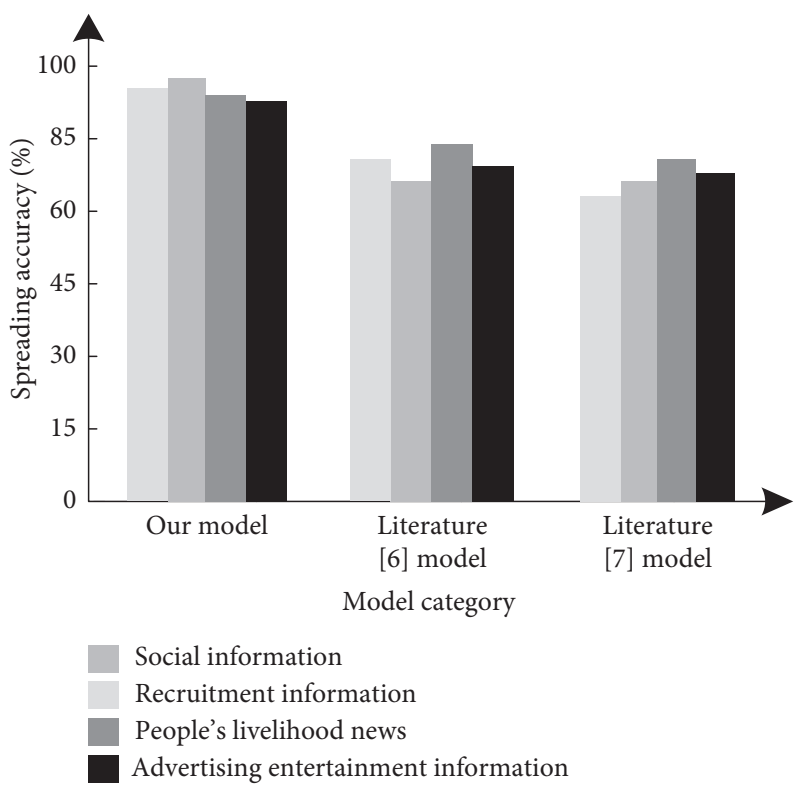

Figure 11: Comparison results of the three models.

TABLE 2: Information dissemination fitting results of the three models (piece).

\begin{tabular}{lcccc}
\hline Fitting point & Our model & Reference [6] model & Reference [7] model & The actual dissemination \\
\hline 1 & 0 & 0 & 0 & 0 \\
2 & 22 & 18 & 20 & 23 \\
3 & 1800 & 120 & 996 & 1000 \\
4 & 1896 & 1850 & 1275 & 1330 \\
5 & 1720 & 2000 & 1356 & 2010 \\
6 & 1998 & 2500 & 940 & 1510 \\
7 & 1998 & 3220 & 775 & 1760 \\
8 & 1500 & 3540 & 502 & 1505 \\
9 & 1255 & 3368 & 495 & 1500 \\
10 & 1120 & 2250 & 480 & 1005 \\
11 & 805 & 1510 & 468 & 262 \\
12 & 256 & 1022 & 202 & 246 \\
\hline
\end{tabular}

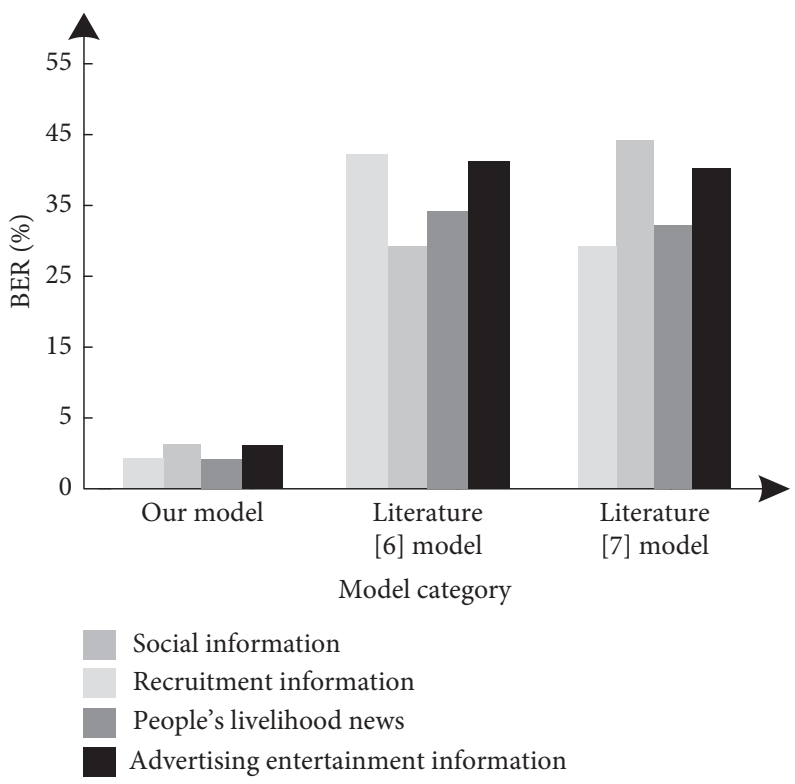

FIGURE 12: Input bit error rate test results of the three models. 


\section{Conclusion}

Media information dissemination in a wireless network is affected by many factors. The study of factors affecting information dissemination is helpful to understand the law of information dissemination. It is of great significance to the control of information dissemination. This paper studies the media information dissemination model based on a wireless network, which is used for media information dissemination prediction. The model considers the influence of the interaction between nodes on information dissemination and can properly describe the influence of nodes in information dissemination. By optimizing the deep residual network, the information dissemination in a practical application can be better realized. The test results show that this model can accurately realize the classification of different types of media information, and the average error and error rate of information dissemination is low. Therefore, it can reflect the characteristics of media information dissemination in a wireless network in practical application to a large extent. It can accurately predict the status of media information dissemination in a wireless network.

\section{Data Availability}

The datasets used and/or analyzed during the current study are available from the corresponding author on reasonable request.

\section{Conflicts of Interest}

The authors declare that they have no conflicts of interest.

\section{References}

[1] T. K. T. Ho, Q. V. Bui, and M. Bui, "Information diffusion on complex networks: a novel approach based on topic modeling and pretopology theory," Vietnam Journal of Computer Science, vol. 6, no. 3, pp. 285-309, 2019.

[2] A. Hamad and A. D. Jasim, "ECG signal classification based on deep learning by using convolutional neural network (CNN)," Iraqi Journal of Information \& Communications Technology, vol. 3, no. 3, pp. 12-23, 2020.

[3] A. Zappone, M. Di Renzo, and M. Debbah, "Wireless networks design in the era of deep learning: model-based, AIbased, or both?" IEEE Transactions on Communications, vol. 67, no. 10, pp. 7331-7376, 2019.

[4] B. Priambodo and A. Ahmad, "Traffic flow prediction model based on neighbouring roads using neural network and multiple regression," Journal of Information and Communication Technology, vol. 17, no. 4, pp. 513-535, 2018.

[5] W. Shen and X. Li, "Facial expression recognition based on bidirectional gated recurrent units within deep residual network," International Journal of Intelligent Computing and Cybernetics, vol. 13, no. 4, pp. 527-543, 2020.

[6] Y. Zhang and K. He, "Information dissemination model with improved probability based on influence of neighbors for social network," Application Research of Computers, vol. 35, no. 3, pp. 755-759+764, 2018.

[7] X. M. Han, "Study on WeChat moments information dissemination model based on analysis of user behavior," Modern Information, vol. 38, no. 7, pp. 62-66, 2018.
[8] H. Du, G. Wang, and J. Li, "Transformer fault identification with an IF-1DCNN based on informative integration of heterogeneous sources," Mathematical Problems in Engineering, vol. 2021, Article ID 6648919, 14 pages, 2021.

[9] Y. Chi and Y. Liu, "Research on online and offline public opinion evolution model based on the theory of supernetwork," System Engineering Theory and Practice, vol. 39, no. 1, pp. 259-272, 2019.

[10] X. Lin, Q. Jiao, and L. Wang, "Information propagation over networks with antagonistic interactions: the equilibrium analysis," IEEE Transactions on Control of Network Systems, vol. 7, no. 2, pp. 592-602, 2020.

[11] M. Li, Q. Li, Y. Li, Y. Cui, X. Zhao, and L. Guo, "Analysis of characteristics of tennis singles matches based on $5 \mathrm{G}$ and data mining technology," Security and Communication Networks, vol. 2021, no. 20, pp. 1-9, 2021.

[12] J. Li, C. Qu, S. Peng, and Y. Jiang, "Ship detection in sar images based on generative adversarial network and online hard examples mining," Journal of Electronics and Information Technology, vol. 41, no. 1, pp. 143-149, 2019.

[13] N. Kumar and D. Kumar, "An improved grey wolf optimization-based learning of artificial neural network for medical data classification," Journal of Information and Communication Technology, vol. 20, no. 2, pp. 213-248, 2021.

[14] J. Yang, Y. Liu, Z. Liu, Y. Wu, T. Li, and Y. Yang, "A framework for human activity recognition based on WiFi CSI signal enhancement," International Journal of Antennas and Propagation, vol. 2021, Article ID 6654752, 18 pages, 2021.

[15] Z. W. Lan, D. Ji, S. M. Wang, T. L. Lu, and J. Meng, "Identification of curcumin content in raw and vinegarprocessed rhizomes of Curcuma kwangsiensis based on electronic nose combined with back dissemination neural network," China Journal of Chinese Materia Medica, vol. 45, no. 16, pp. 3863-3870, 2020.

[16] J. Dong, W. Qin, Y. Li, Q. Li, and L. Deng, "Fast multiobjective antenna design based on improved back dissemination neural network surrogate model," Journal of Electronics and Information Technology, vol. 40, no. 11, pp. 2712-2719, 2018.

[17] W. E. Suherman, A. H. Rambe, N. Mubarakah, and M. AlAkaidi, "Underground object detection based on radio dissemination characteristics," Journal of Theoretical and Applied Information Technology, vol. 99, no. 1, pp. 48-58, 2021.

[18] M. D. Migliore, "Who cares about the horse? A gentle introduction to information in electromagnetic theory [wireless corner]," IEEE Antennas and Propagation Magazine, vol. 62, no. 5, pp. 126-137, 2020.

[19] R. Gaeta, "A model of information diffusion in interconnected online social networks," ACM Transactions on the Web, vol. 12, no. 2, pp. 1-21, 2018.

[20] J. N. Sun and C. Zhao, "Simulation of communication security frequency domain equalization method based on energy optimization," Computer Simulation, vol. 37, no. 3, pp. 421-424+457, 2020. 